\title{
Chief Complaint: Ingested Button Battery
}

\author{
David Rayburn, MD, MPH \\ Julie Welch, MD \\ Emergency Medicine-Pediatrics, Indiana University \\ 1701 N. Senate Blvd. AG012, \\ Indianapolis, IN 46202
}

\section{Visual Care Discussion}

A 3-year-old healthy female was brought to the ED for the potential ingestion of a foreign body (FB), thought to be a button battery (Fig. 1). The child was acting normally and complained of itching in her throat. On examination, her vital signs were stable. She was in no acute distress. Oral pharynx was clear and voice was normal. Respirations were clear with no airway stridor. Abdomen was soft and non-tender.

Evaluation included a CXR and KUB to determine the location of the FB. CXR was clear without evidence of FB (Fig. 2). The KUB, however, demonstrated a circular FB located in the stomach, pre-pyloric, and just one vertebral body lower than was visible on the CXR (Fig. 3).

The FB in the stomach was presumed to be a button battery; therefore pediatric general surgery was consulted. When the battery is located in the stomach there is the option to attempt endoscopic retrieval or observe with serial radiographs. In this case, it was decided to observe the patient.

This is the author's manuscript of the article published in final edited form as: 
Figure 1. Skil keychain flashlight with paperclip for size comparison. Flashlight contained a standard button battery, which was absent when opened in the ED.

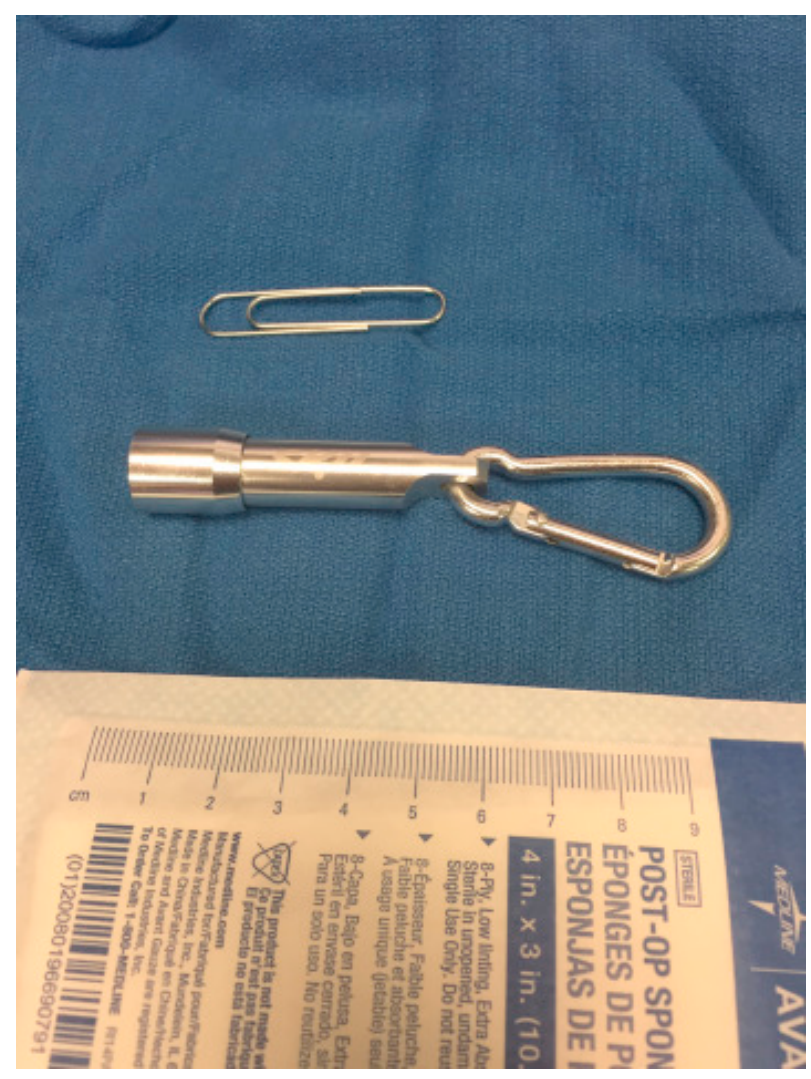

Figure 2. Patient's CXR with no evidence of foreign body or button battery.

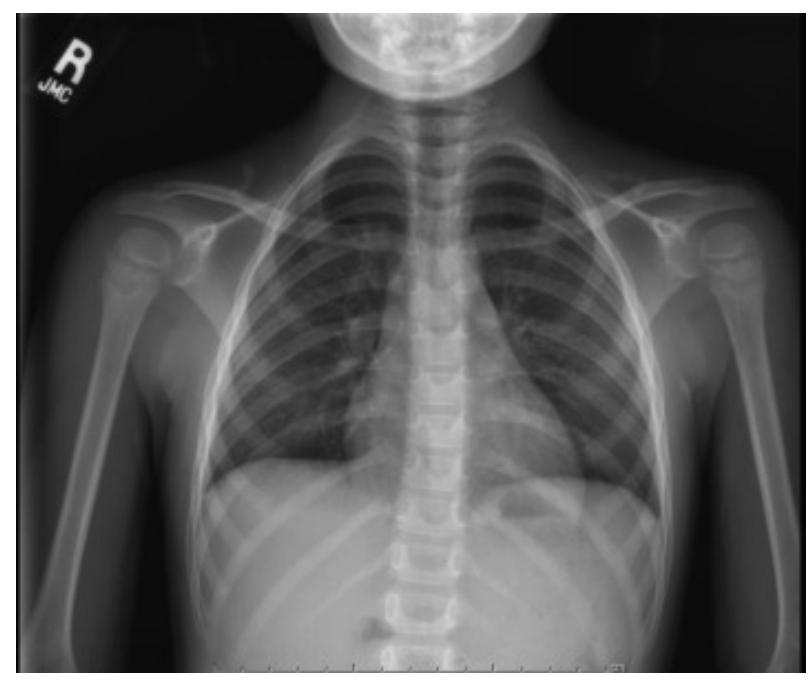


Figure 3. Patient's KUB demonstrating a foreign body, button battery, located in the stomach with no evidence of free air.

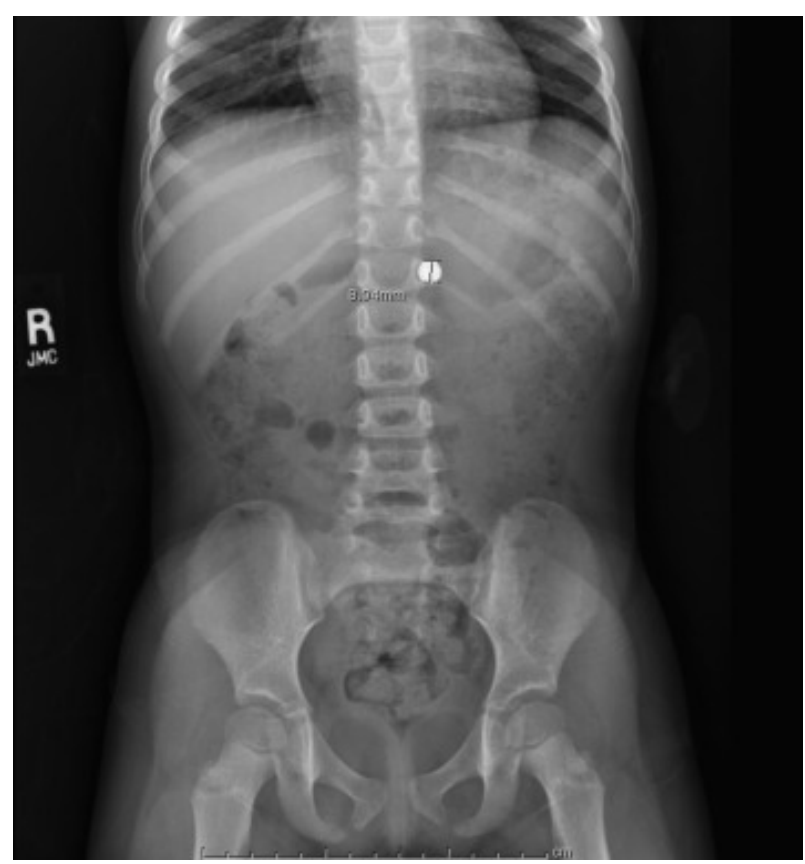

